\title{
In-situ X-ray study of phase transformations in Ti-Nb-based SMA under variable stress-temperature conditions: Preliminary results
}

\author{
DUBINSKIY Sergey ${ }^{1,2, a}$, BRAILOVSKI Vladimir ${ }^{1, b}$, PROKOSHKIN Sergey ${ }^{2, \mathrm{c}}$, \\ INAEKYAN Karine ${ }^{1, d}$
}

\author{
${ }^{1}$ Ecole de technologie supérieure, 1100, Notre-Dame Str. West, Montreal (Quebec), H3C \\ $1 \mathrm{~K} 3$, Canada \\ ${ }^{2}$ National University of Science and Technology "MISIS", 4, Leninskiy prosp., Moscow \\ 119049, Russian Federation \\ asdubinskiy@gmail.com, ${ }^{b}$ vladimir.brailovski@etsmtl.ca, ${ }^{c}$ prokoshkin@tmo.misis.ru, \\ dkarine.inaekyan@etsmtl.ca,
}

Keywords: Titanium alloys, shape memory alloys, X-ray diffraction analysis, in-situ tensile stage

\begin{abstract}
The technique and preliminary results of in situ X-ray diffraction analysis of the martensitic transformation in the newly developed Ti-Nb-Zr SMA for biomedical application are presented. To perform the in situ analysis, an original tensile stage, powered by a Ti-Ni SMA actuator and fit within the "TTK450" thermal chamber of a "PANalytical X'Pert Pro" diffractometer is designed, manufactured and validated. The tensile stage working principle and analysis methodology are described in detail. Preliminary results obtained during in-situ X-ray analysis of the phase transformations in Ti-Nb-Zr SMA are also presented.
\end{abstract}

\section{Introduction}

High elastic modulus and linear stress-strain behaviour with limited reversible strain of conventional metallic implant materials' compromise their biomechanical compatibility. Ti-Ni shape memory alloys (SMA) are better suited for this role because of their superelasticity which closely mimicks bone behaviour [1,2], but their application in medicine is hindered by the presence of toxic nickel [3]. A new generation of multicomponent $\mathrm{Ni}$-free Ti-Nb-based metastable $\beta$-titanium alloys containing only non-toxic elements ( $\mathrm{Ta}, \mathrm{Zr}, \mathrm{Mo}, \mathrm{O}$ ) [4,5] is emerging, offering the most promising candidates for the role of metallic implant materials. Shape memory and superelasticity observed in these alloys are due to thermoelastic $\beta \leftrightarrow \alpha$ " martensitic transformation. Since $\mathrm{Ti}-\mathrm{Nb}$ based SMA are multiphase alloys, they generally contain, in addition to body centered cubic $\beta$-“austenite" and orthorhombic $\alpha$ "-martensite, hexagonal $\alpha, \alpha$ '-martensite and $\omega$-phase. That significantly complicates their microstructural analysis and therefore the understanding of the features of low-temperature phase transformations in these materials.

This paper is focused on in situ X-ray diffraction study of phase transformations in Ti-Nb-Zr SMA under variable stress-strain-temperature conditions. This work addresses the design, manufacture and validation of an original tensile stage lodged within the "TTK450" thermal chamber of a "PANalytical X'Pert PRO" diffractometer and powered by Ti-Ni SMA actuator. Preliminary results obtained during in situ X-ray diffraction analysis of the structure and phase changes under stress in Ti-Nb-Zr SMA are also presented.

\section{Experimental}

Ti-Nb-Zr alloy samples are used in this work. A Ti-21.8Nb-6Zr (at.\%) cylindrical ingot (50 mm diameter, $660 \mathrm{~mm}$ long) was produced by induction skull melting ("Flowserve Corp.", USA) and subjected to hot isostatic pressing $\left(900^{\circ} \mathrm{C}, 100 \mathrm{MPa}, 2 \mathrm{~h}\right)$. For X-ray analysis, $1.2 \times 0.8 \times 100 \mathrm{~mm}$ plates were EDM-cut from the ingot, cold-rolled (logarithmic thickness reduction $e=0.37$ ) and annealed $\left(600^{\circ} \mathrm{C}, 0.5 \mathrm{~h}\right)$. These processing conditions are based on the results obtained in [6]. 
After thermomechanical processing, the plates were cut into $30 \mathrm{~mm}$-long samples and mechanically grinded, polished and chemically etched to $0.1 \mathrm{~mm}$ thickness.

The tensile stage mechanism shown in Fig.1 is especially designed for use with SMA actuators. As a consequence, it can be integrated in a "TTK450" thermal chamber. The SMA actuator makes it possible to use the stage in the $-196 \ldots+150^{\circ} \mathrm{C}$ temperature range. The maximum force which can be applied to the sample is $250 \mathrm{~N}$, maximum elongation is $2 \mathrm{~mm}$, the minimum sample working length is $16.5 \mathrm{~mm}$. and the recommended exposed sample area for analysis is $3.5 \mathrm{~mm} \times 1.5 \mathrm{~mm}$.

Fig. 1 illustrates the tensile stage mechanism. The main frame (1) provides rigidity to the stage and holds the pivot axes (2) of the rotating levers (3) transmitting the tensile force from the SMA actuator (4) to the specimen (5). The actuator-to-specimen levers length ratio can be adjusted from $1: 1.5$ to $1: 2$.

To avoid X-ray defocusing during scanning, the guiding shafts (6) maintain the specimen (5) in a constant vertical position. To electrically isolate the SMA actuator (4), polymer sleeves are used as insulators between the actuator tilting grip system (7) and the levers (3). The identical tilting grip system (7) is used for specimen (5) fixation. The glass plate (8) is used to avoid X-ray diffraction from the main frame (1). All the parts except the insulator sleeves are made of stainless steel.

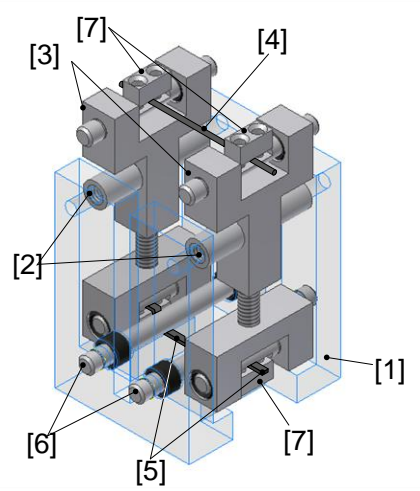

a)

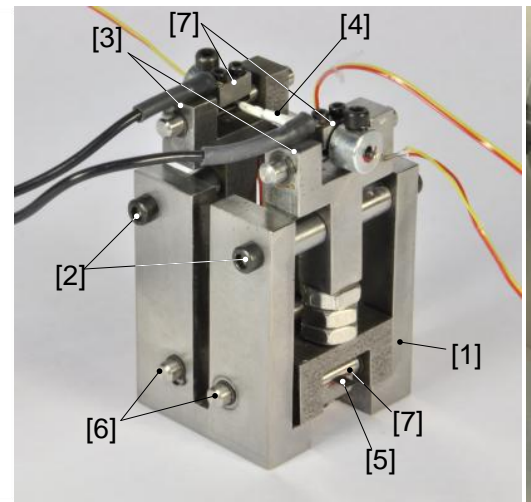

b)

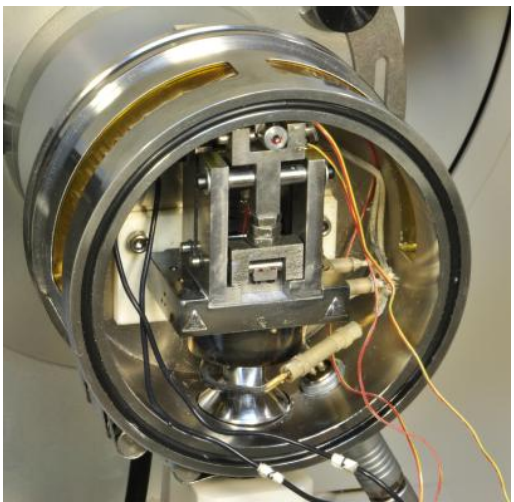

c)

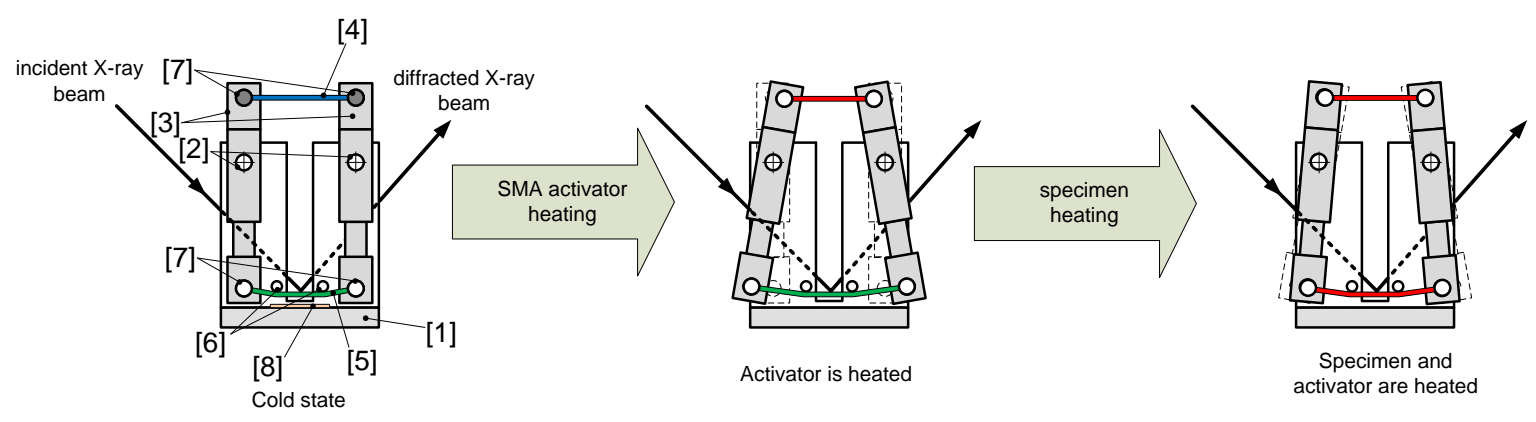

d)

Fig.1. Tensile stage mechanism: a) 3D prototype computer model; b) photo of the tensile stage mechanism; c) tensile stage inside a TTK450 thermal chamber; d) operation principle

The main frame (1) is directly mounted on the thermo-control plate of the thermal chamber, and a thin layer of silicone grease is applied to both connected surfaces to improve heat transfer from the control plate of the thermal chamber to the specimen; boron nitride powder is used as a thermoconductive lubricant for all the rubbing elements.

The operation principle is illustrated in Fig.1d. Initially the actuator (4) and the specimen (5) are connected in their "cold" state. When the actuator is heated, while the specimen is not, the first shortens and stretches the second, and the whole system reaches its second equilibrium position. 
When the specimen is heated in its turn, it also starts to generate recovery stresses, opposing the actuator, and the third equilibrium position is reached.

The SMA actuator represents a $1 \mathrm{~mm}$ diameter Ti-Ni SMA wire ("SAES GETTERS") with an active length of $16.3 \mathrm{~mm}$. Before installation, the Ti-Ni wire was subjected to thermomechanical cycling under constant stress to induce and stabilize a two-way shape memory effect in the material. This wire actuator generates either recovery stresses up to $\sigma_{\mathrm{r}}{ }^{\max }=530 \mathrm{MPa}$ or recovery strains up to $\varepsilon_{\mathrm{r}}^{\max }=3 \%$. These recovery stress-strain characteristics of the Ti-Ni active element (4) correspond to the $\sim 415 \mathrm{~N}-0.5 \mathrm{~mm}$ force-stroke characteristics of the SMA actuator. Considering a $1: 2$ actuatorspecimen levers length ratio, $208 \mathrm{~N}-1 \mathrm{~mm}$ force - stroke loading can be applied to the sample.

The actuator is heated by direct Joule heating and its temperature is measured by a K-type thermocouple ("TT-K-36-SLE(ROHS), Omega"), insulated and fixed to the actuator. For thermocouple reading, a USB data acquisition module ("NI USB-6211", "Nat'l Instr.") is used. Lab-View software with a PID controller ("LabView 9.0", "Nat'l Instr.") and a programmable power supply ("SPS100-33-KOU2", "American Reliance”) allow for control of the SMA actuator temperature.

The $-150^{\circ} \mathrm{C} \ldots+100^{\circ} \mathrm{C}$ temperature range used in our in situ X-ray structure analysis encompasses the major structural changes related to the $\beta \leftrightarrow \alpha$ " martensitic transformations observed in the studied Ti-21.8Nb-6Zr (at.\%) alloy [6].The TTK thermal chamber with the miniature tensile stage developed for this study is installed in the "PANalytical X'Pert Pro" diffractometer with $\mathrm{Cu}_{\mathrm{K} \alpha}$ monochromatized radiation. Three X-ray analysis sequences are schematically represented in Fig. 2. The first (reference) sequence (stress-free, SF) of temperature scanning (Fig. 2a), implies cooling of the non-stressed sample down to $-150^{\circ} \mathrm{C}(1-2)$ followed by heating it up to $100^{\circ} \mathrm{C}(2-3)$. The second and the third sequences both imply temperature scanning under a constant strain. When the specimen is loaded in its austenitic state (room temperature), this sequence is called the "constantstrain-from-austenite, CS(A)" temperature scanning (Fig. 2b). When the specimen is loaded in its martensitic state, this sequence is called the "constant-strain-from-martensite, CS(M)" temperature scanning (Fig. 2c). The CS(A) and CS(M) X-ray study sequences allow analysis of both the temperature- and the strain-induced direct and reverse martensitic transformations and reversible martensite reorientation.
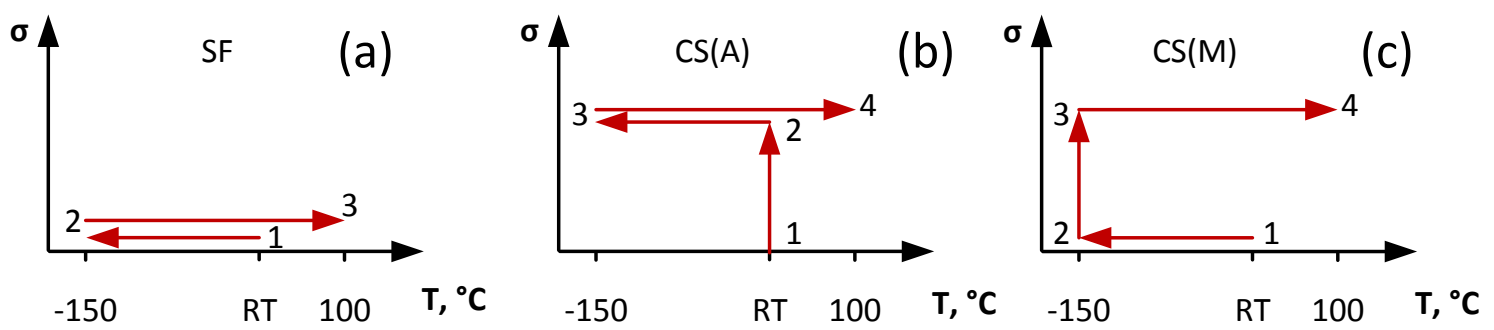

Fig.2. In situ X-ray study sequences: a) stress-free (SF) temperature scanning; constant-strain temperature scanning with loading in b) austenitic $\mathrm{CS}(\mathrm{A})$ and c) martensitic $\mathrm{CS}(\mathrm{M})$ states.

\section{Results and Discussion}

This work is limited to the presentation of some preliminary results obtained using SF and $\mathrm{CS}(\mathrm{M})$ schedules. Fig. 3a shows X-ray diffractograms corresponding to RT, $-110^{\circ} \mathrm{C}$ and $+100^{\circ} \mathrm{C}$ temperatures (SF) in a consecutive order. It can be observed that $\beta$-austenite lines $\{110\},\{200\}$ $\{211\},\{220\}$ and $\{310\}$ are present at all the X-ray scanning temperatures, whereas $\alpha$ ''-martensite lines (020), (111), (012), (102), (200), (130) and (131) appear when the specimen is cooled down and disappear when it is heated again, being accompanied with the X-ray lines of "athermal" $\omega$-phase appearance and disappearance. From these diffractograms, it is also possible to measure thermal expansion/contraction strains $(\Delta d / d)_{h k l}$. 
In the insert to Fig. 3a, a relative variation of the interplanar distance corresponding to the $110_{\beta}$ peak $(\Delta d / d)_{110}$ is plotted as a function of the temperature: stress-free cooling from RT to $-150^{\circ} \mathrm{C}$ (path $1 \rightarrow 2$, one scan at RT) and stress-free heating from -150 to $+100^{\circ} \mathrm{C}$ (path $2 \rightarrow 3,8$ scans). For the SF sequence, $(\Delta d / d)_{110}$ values are equal to the thermal contraction/expansion strain, $\left(\varepsilon_{\Delta T}\right)_{110}$; and they are calculated from the angular shifts of the $110_{\beta}$ peak position.

On heating (path $2 \rightarrow 3$ ), a net deviation from the linear behaviour can be observed, starting at about $-75^{\circ} \mathrm{C}$, thus pointing to the onset of $\alpha " \rightarrow \beta$ and $\omega \rightarrow \beta$ phase transformations.
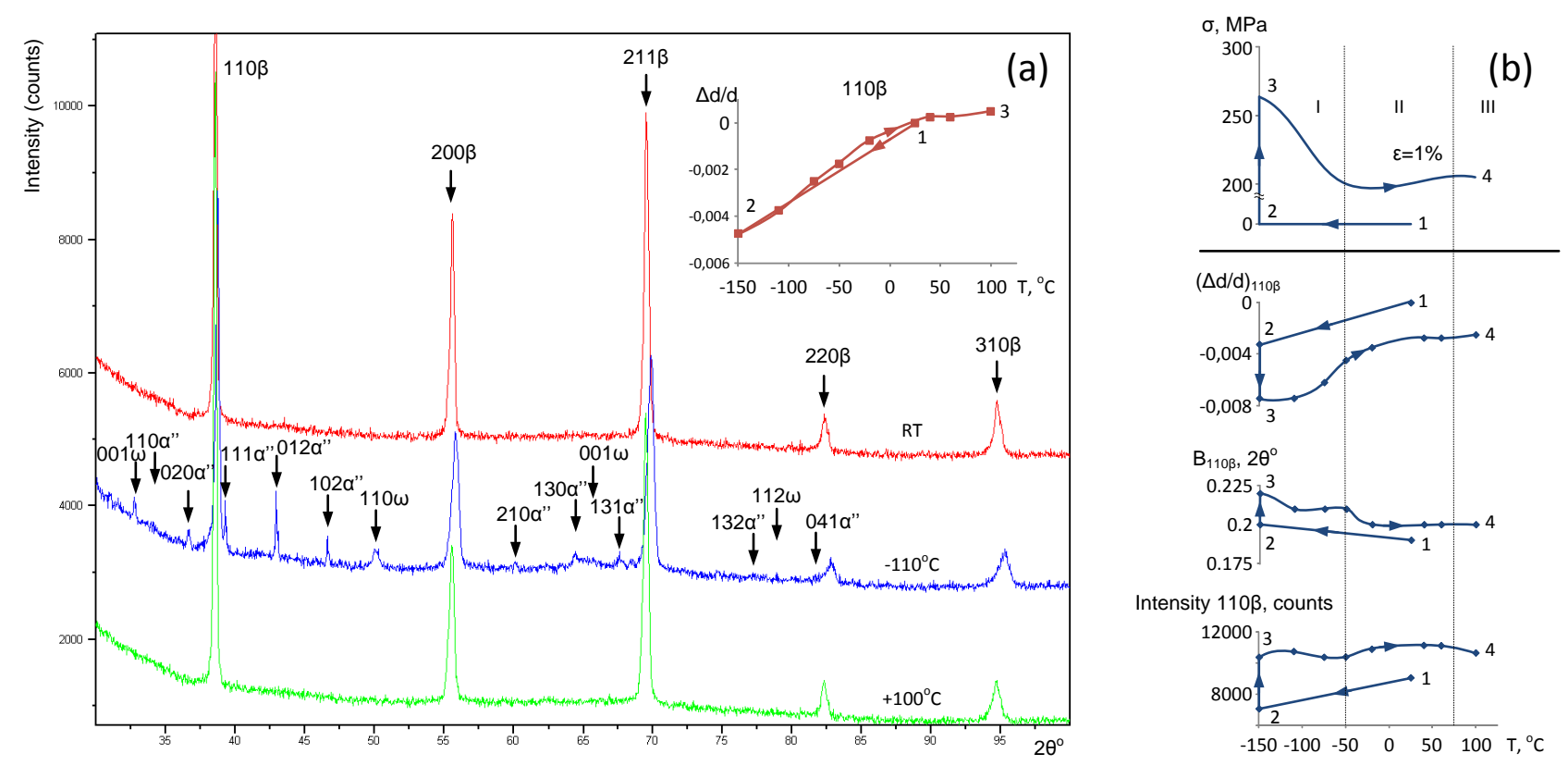

Fig. 3. Ti-21.8Nb-6Zr (at.\%) alloy: a) SF sequence: X-ray diffraction profiles for selected temperatures and relative variation of the $110_{\beta}$ interplanar distance as a function of temperature (insert); b) CS(M) sequence: $110_{\beta} \mathrm{X}$-ray line parameters' variation as functions of the temperature.

In Fig. $3 b$, the $110_{\beta}$ line parameters measured during the $\operatorname{CS}(\mathrm{M})$ sequence are plotted as functions of the temperature. These parameters comprise: the relative variation of the interplanar distance $(\Delta d / d)_{110}$ obtained from the angular shift of the peak position, the peak mid-height width $\left(B_{110}\right)$ and the intensity (peak height) $\left(I_{110}\right)$. For the sake of comparison, a stress-temperature diagram obtained during previous constant-strain temperature scanning experiments is also plotted in Fig. 3b [6]. This last test was realized under $1 \%$ constant strain using exactly the same CS(M) sequence.

In Fig. 3b, SC(M) sequence, path 1-2 of all the plots corresponds to cooling from RT to $-150^{\circ} \mathrm{C}$, path 2-3 to stretching at $-150^{\circ} \mathrm{C}$, and path 3-4 to heating under a constant strain. Three temperature ranges can be distinguished in the heating path 3-4: the first range, $-150^{\circ} \mathrm{C} \ldots-50^{\circ} \mathrm{C}$, corresponding to stress relaxation caused by thermal expansion, the second interval, $-50^{\circ} \mathrm{C} \ldots+75^{\circ} \mathrm{C}$, where the recovery stresses generated in course of $\alpha " \rightarrow \beta$ transformation overcome the stress relaxation caused by thermal expansion, and the third range, $>75^{\circ} \mathrm{C}$, where the stress relaxation due to thermal expansion exceeds the stress generated due to constrained reverse martensitic transformation.

Considering that the X-ray strain analysis is based on the measurement of variations of interplanar distances in the direction perpendicular to the specimen surface (transverse direction), and that these variations contain two components: the first related to thermal contraction/expansion and the second related to the applied external loads, the total transverse strain measured by X-ray diffraction can be written as $\Sigma \varepsilon_{\perp}=\varepsilon_{\Delta T}+\varepsilon_{\perp}$, where $\varepsilon_{\Delta T}$ is the thermal expansion strain known from the previous SF mode measurements, and $\varepsilon_{\perp}$ is the transverse strain caused by external load, all 
three corresponding to a given X-ray scanning temperature. From the transverse strain caused by external load, it is possible to evaluate a longitudinal tensile strain $\varepsilon$ applied to the sample. Fig. 4 illustrates the conversion from the measured transverse strain (Fig. 4a) to the longitudinal tensile strain applied to the specimen (Fig. 4c). This conversion is made by subtracting the thermal expansion strain (Fig.4b) and dividing the result obtained by the Poisson's ratio. It can be seen from Fig. $4 \mathrm{c}$ that the real strain applied to the specimen when heated from -150 to $+100^{\circ} \mathrm{C}$ is not constant. It varies between 0.82 to $1.2 \%$, with an average value of $1 \%$. These variations are caused by a nonuniform temperature distribution in the tensile stage and its limited rigidity.
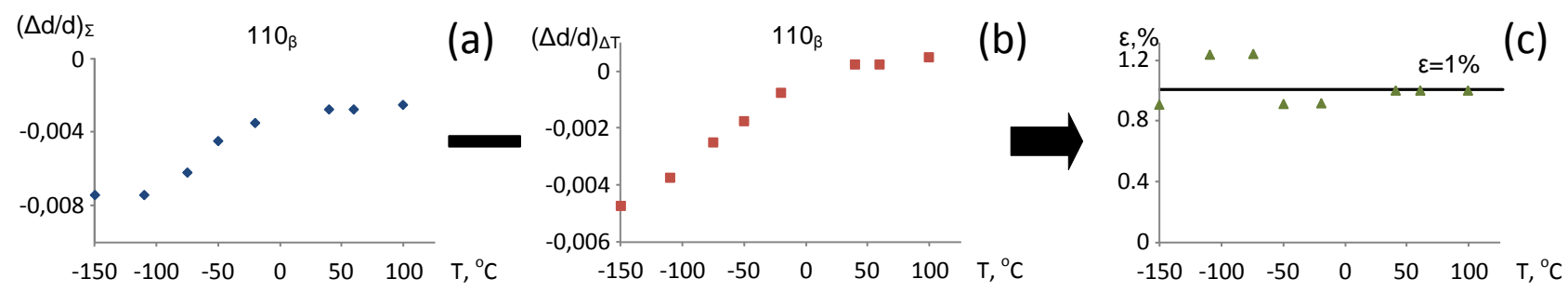

Fig.4. a) Total transverse strain (CS(M) sequence), b) thermal expansion strain, (SF sequence), and c) longitudinal tensile strain applied to the specimen (all the measurements are made on heating).

\section{Summary}

An original tensile stage fitted within the TTK450 thermal chamber of a "PANalytical X'Pert PRO" diffractometer and powered by a Ti-Ni SMA actuator is designed, manufactured and successfully validated. Three sequences of in situ X-ray martensitic transformation analysis are realized: stressfree temperature scanning, constant-stress temperature scanning with loading in the austenitic state and constant-stress temperature scanning with loading in the martensitic state. Preliminary results obtained for biomedical Ti-Nb-Zr SMA by means of in-situ X-ray analysis under variable forcetemperature conditions are presented and analysed.

\section{Acknowledgements}

The authors are grateful to the Natural Science Engineering Research Council of Canada and the Ministry of Education and Science of the Russian Federation for the financial support.

\section{References}

[1] J. Ryhänen, Biocompatibility Evaluation of Nickel-Titanium Shape Memory Alloy, Academic Dissertation, University of Oulu, 1999.

[2] T. Yoneyama, S. Miyazaki, Shape Memory Alloys for Biomedical Applications, Woodhead Publishing Ltd, England (2010).

[3] K. Takamura, K. Hayashi, N. Ishinishi, T. Yamada, Y. Sugioka, Evaluation of carcinogenicity and chronic toxicity associated with orthopedic implants in mice, J. Biomed. Mater.Res. 28 (1980) 583-589.

[4] S. Miyazaki, H.Y. Kim, H. Hosoda, Development and characterization of Ni-Free Ti-based shape memory and superelastic alloys, Mater. Sci. Eng. A. 438-440 (2006) 18-24.

[5] E.W. Collings, Titanium alloys, A.S.f. Metals, 1984.

[6] V. Brailovski, S. Prokoshkin, K. Inaekyan, S. Dubinskiy, M. Gauthier, Mechanical properties of thermomechanically processed metastable beta $\mathrm{Ti}-\mathrm{Nb}-\mathrm{Zr}$ alloys for biomedical applications, Mater. Sci. Forum 706-709 (2012) 455-460. 